\title{
Necrotizing Fasciitis due to Perforated Appendix
}

\author{
Bryan P. Mosher ${ }^{1}$, Mark T. Kowal ${ }^{1,2}$ \\ University of Washington ${ }^{1}$ School of Medicine, University of Washington ${ }^{2}$ Department of Surgery, Seattle, WA 98195.
}

\section{Corresponding Author: \\ Bryan P. Mosher}

Email: bpmosher@uw.edu

This is an Open Access article distributed under the terms of the Creative Commons Attribution License (creativecommons.org/ licenses/by/3.0).

Received

Accepted

August 1, 2017

Published

October 17, 2017

November 25, 2017

\begin{abstract}
Background: Appendectomies are routine procedures done with minimal complications. However, the diagnosis of acute appendicitis may be complicated by its' varied presentations. Although extremely rare, a perforated appendix is capable of causing the often fatal necrotizing fasciitis infection. Case Report: A 42-year-old hypotensive, tachycardic female with acute abdominal pain was brought to the operating room after CT findings were suspicious for acute appendicitis. Laparoscopic dissection revealed that the appendix had eroded through the abdominal wall, potentially through a hernia defect or a defect created by the infection and resulting inflammation. The appendix was removed and a drain was left in the right pericolic gutter. The patient was repositioned and a 10 $\mathrm{cm}$ incision was made in the right flank where a large amount of necrotic foul-smelling tissue and fluid was removed. Bacterial culture of the pus grew Peptostreptococcus and Escherichia coli. After clearing a significant amount of necrotic tissue and receiving a bedside consultation from orthopedics, the wound was packed. Conclusion: Necrotizing fasciitis is a rare, serious disease that is capable of, although very rarely, being caused by a perforated appendix. The high mortality rate associated with necrotizing fasciitis caused by appendicitis highlights the need for early diagnosis, broad-spectrum antibiotic treatment as well as timely surgical debridement to maximize chance of survival.
\end{abstract}

Keywords: Abdominal Pain, Appendicitis, Drainage, Necrotizing Fascitis, Tomography.

\section{Introduction}

Appendectomy for acute appendicitis is the most common urgent intra-abdominal surgical procedure performed and is done routinely with minimal complications [1]. Despite this, appropriate diagnosis of acute appendicitis can at times be complicated by the varied presentations with which it arose. If a proper diagnosis is not made in a timely manner the appendix may perforate which is associated with increased morbidity and mortality [2]. Necrotizing fasciitis is an uncommon potentially fatal soft tissue infection that is usually caused by toxin-producing bacteria and is associated with high mortality, upwards of $76 \%$ [3]. Despite needing to consider acute appendicitis in the etiology of necrotizing fasciitis, it is extremely rare and often fatal [4]. We report a case of necrotizing fasciitis of the right flank secondary to a perforated appendix.

\section{Case Report}

A 42-year-old female with a medical history of obesity, borderline diabetes, depression, alcohol and cocaine use disorders and anemia presented to our emergency department with abdominal pain after sustaining multiple falls 3-4 days back while shoveling snow. On admission her temperature was $36.6^{\circ} \mathrm{C}$, blood pressure was $94 / 52 \mathrm{mmHg}$ and heart rate was 128 beats/minute. She was oriented to person, place and time. Her physical examination revealed a soft abdomen that was diffusely tender to palpation, but primarily on the right side. There 
was also a warm, firm, tender collection along the right flank that extended towards the right iliac crest.

Initial laboratory results revealed a white blood cell count of $14.0 \times 10^{3} / \mu \mathrm{L}$ with $83 \%$ neutrophils. Her hemoglobin was $5.7 \mathrm{~g} / \mathrm{dL}$ and hematocrit was $16.5 \%$ volume. Her creatinine was $1.12 \mathrm{mg} / \mathrm{dL}$, sodium was $131 \mathrm{mmol} / \mathrm{L}$ and potassium was $2.6 \mathrm{mmol} / \mathrm{L}$. Urine toxicology was positive for cocaine. Abdominal computed tomography (CT) scan done in the emergency department revealed a large transfascial right retroperitoneal and flank subcutaneous collection containing a significant amount of gas within it [Fig.1]. There was also a small amount of free fluid in the right lower quadrant. The appendix was not well-delineated and involvement of the right colon was uncertain. However, the right and proximal transverse colon walls were diffusely thickened with marked retrocecal inflammatory changes along with several small adjacent extra-luminal air bubbles, raising the question of appendiceal and/ or colonic involvement. Thus perforated retrocecal appendicitis was a consideration on the differential along with necrotizing fasciitis.

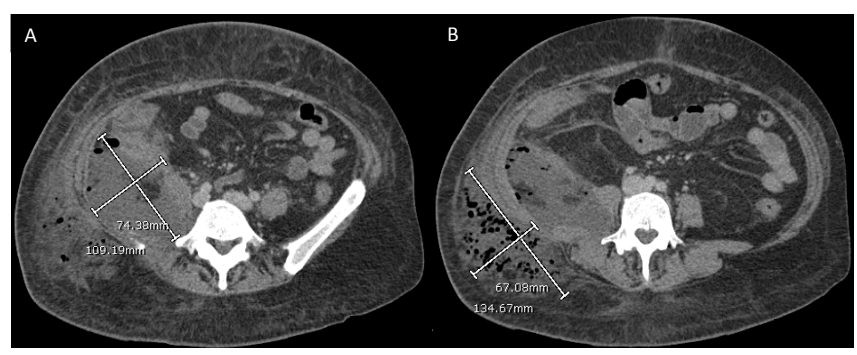

Fig.1: Selected axial views of the abdominal CT scan (a): Complex, partially loculated air-containing right retroperitoneal collection displacing the right colon, terminal ileum and right kidney antero-medially measuring $7.4 \times 10.9$ $x 16.1 \mathrm{~cm} W$ by $A P$ by $C C$ (b): There is an associated $2 \times 3$ $x 8 \mathrm{~cm}$ fusiform partially loculated right iliopsoas collection with the dominant collection communicating through a tiny defect in right postero-lateral abdominal wall musculature with an additional poorly loculated, extensive air-containing right postero-lateral flank subcutaneous soft tissue collection measuring $6.7 \times 13.5 \times 15.0 \mathrm{~cm} W$ by $A P$ by $C C$. There is extensive stranding in adjacent soft tissues and the right abdominal wall musculature is thickened.
After transfusing the patient with four units of packed red blood cells and starting intravenous piperacillin/tazobactam, vancomycin and clindamycin, the patient was urgently taken to the operating room. Diagnostic laparoscopy revealed an appendix that was completely adhered to the right abdominal wall [Fig.2]. Dissection revealed that the appendix had eroded through the abdominal wall, potentially through a hernia defect or a defect created by the infection and resulting inflammation. The appendix was then removed and a drain was left in the right pericolic gutter. The patient was then repositioned and a $10 \mathrm{~cm}$ incision was made in the right flank where a large amount of necrotic foul-smelling tissue and fluid was removed. Bacterial culture of the pus grew Peptostreptococcus and Escherichia coli. This extended cephalad towards the $12^{\text {th }}$ rib, inferiorly toward the iliac crest, posteriorly towards the paraspinous muscles and anteriorly into the peritoneal space. The right flank drainage area was able to be connected by finger dissection to the intraperitoneal space and the drain that was left at the time of appendectomy could be palpated. After clearing a significant amount of necrotic tissue and receiving a bedside consultation from orthopedics, the wound was packed with quarter strength Dakin's soaked Kerlix. The patient was hemodynamically stable throughout her operation, but did receive two additional units of packed red blood cells while in the operating room. A central line in the right internal jugular vein and a right-sided arterial line were placed by anesthesia prior to going to the intensive care unit (ICU). Plans were quickly made to Medevac the patient to the regional trauma

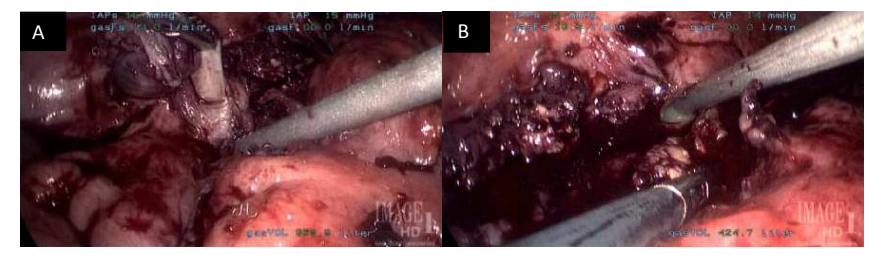

Fig.2: Intra-operative laparoscopic images (a): Appendix being removed from abdominal wall. (b): After removal of the appendix from the abdominal wall, a hole can be seen filled with yellow necrotic liquid which was aspirated. 
center to allow for more appropriate care given the need for additional surgeries as well as services not available at our hospital. At time of discharge, the patient was hemodynamically stable, producing urine and her labs were beginning to normalize.

\section{Discussion}

We present the case of a 42-year-old female who presented to the emergency department with abdominal pain after sustaining multiple falls 3-4 days before. On admission she was tachycardic and her abdomen was soft and diffusely tender to palpation, primarily on the right side. There was also a warm, firm, tender collection along the right flank that extended towards the right iliac crest. In addition she had an elevated white blood cell count and was anemic. An abdominal CT demonstrated a large trans-fascial right retroperitoneal and flank subcutaneous collection containing a significant amount of gas. From imaging the appendix was not clearly visible and involvement with the right colon could not be ruled out. However, the right and proximal transverse colon walls were diffusely thickened with marked retro-cecal inflammatory changes. This observation along with several small adjacent extra-luminal air bubbles raised the question of appendiceal and/or colonic involvement and perforated retrocecal appendicitis was a consideration on the differential along with necrotizing fasciitis.

In the operating room a diagnostic laparoscopy revealed an appendix that had completely adhered to and eroded through the right abdominal wall. Although the exact causative factors for this migratory pattern is unknown, it could have been through a hernia defect or a defect created by the infection and resulting inflammation. A significant amount of necrotic tissue was removed from the patient's right flank which eventually grew Peptostreptococcus and Escherichia coli on culture.

Necrotizing fasciitis is a rare, serious disease of the body's soft tissues that is most commonly seen in the extremities and perineum [5]. For an infection to occur, subcutaneous tissue must be inoculated with a pathogen. In our patient's case the inoculation was from the ruptured appendix that had adhered to the abdominal wall. After a review of the literature, Takeda et al. concluded that of the 12 published case reports of patients with necrotizing fasciitis caused by appendicitis, four $(30.8 \%)$ of the patients died [6]. This grim statistic highlights the need for early diagnosis, broad-spectrum antibiotic treatment as well as timely surgical debridement if survival is to be achieved. We maintained a level of suspicion about necrotizing fasciitis and started antibiotics in a timely manner which likely increased this patient's chances of survival. Once in the operating room, the thorough removal of necrotic tissue likely also increased chance of survival. Although acute appendicitis may be a common disease, peculiar presentations and complications certainly do arise, such as necrotizing fasciitis, which can be very dangerous if not fatal. If necrotizing fasciitis is found, it is of great importance to act expeditiously to prevent further spread of the disease process.

\section{Conclusion}

Necrotizing fasciitis is a serious disease that carries a high mortality rate. It is important to keep a high level of clinical suspicion when the signs and symptoms are indicative of the infection but the etiology of the disease is uncommon.

Contributors: BPM: manuscript writing, assistant to attending surgeon; MTK: manuscript editing, attending surgeon. BPM will act as guarantor. Both authors approved the final version of this manuscript.

Funding: None; Competing interests: None stated.

\section{References}

1. Körner H, Söndenaa K, Söreide JA, Andersen E, Nysted $\mathrm{A}$, Lende $\mathrm{TH}$, et al. Incidence of acute nonperforated and perforated appendicitis: age-specific and sex-specific analysis. World J Surg. 1997;21:313-317.

2. Papandria D, Goldstein SD, Rhee D, Salazar JH, Arlikar $\mathrm{J}$, Gorgy A, et al. Risk of perforation increases with delay in recognition and surgery for acute appendicitis. J Surg Res. 2013;184:723-729. 
3. McHenry CR, Piotrowski JJ, Petrinic D, Malangoni MA. Determinants of mortality for necrotizing soft-tissue infections. Ann Surg. 1995;221:558-563.

4. Green RJ, Dafoe DC, Raffin TA. Necrotizing fasciitis. Chest. 1996;110:219-229.

5. Hakkarainen TW, Kopari NM, Pham TN, Evans HL. Necrotizing soft tissue infections: Review and current concepts in treatment, systems of care, and outcomes. Curr Probl Surg. 2014;51:344-362.

6. Takeda M, Higashi Y, Shoji T, Hiraide T, Maruo H. Necrotizing fasciitis caused by a primary appendicocutaneous fistula. Surg Today. 2012;42:781784. 